\title{
Network spread of invasive species and infectious diseases
}

\author{
Christopher Strickland ${ }^{\mathrm{a}, 1, *}$, Gerhard Dangelmayr ${ }^{\mathrm{a}}$, Patrick D. Shipman ${ }^{\mathrm{a}}$, \\ Sunil Kumar ${ }^{\mathrm{b}}$, Thomas J. Stohlgren ${ }^{\mathrm{b}}$ \\ ${ }^{a}$ Department of Mathematics, Colorado State University, Fort Collins, CO 80523-1874 \\ ${ }^{b}$ Natural Resource Ecology Laboratory, Colorado State University, Fort Collins, CO \\ 80523-1499
}

\begin{abstract}
Landscape heterogeneity, non-local spreading mechanisms, and long-distance transportation connections can affect the spread of an invasive species or infectious disease. In this paper, we introduce a mathematical model that combines a vector-based transportation network with models for continuous invasive spread. Given a strongly connected, directed graph of transportation rates, we assume that carriers can transport a biological invader to distant sites. Following a possible latent stage, the invader then possibly establishes in the new location and spreads outwards in the continuous domain. Numerical results are shown for the invasion of B. tectorum in Rocky Mountain National Park based on the presence probability model of Strickland et al. (2013, J. Math. Biol. 332, 123-135) and compare favorably with data. Analysis of the network component of the model reveals a unique, stable steady-state solution of the infected vectors.

Keywords: Invasive species, Bromus tectorum, infectious diseases, MaxEnt, niche modeling, species distribution modeling 2000 MSC: 92D25, 60G99, 92D40, 92D30

\footnotetext{
${ }^{*}$ Corresponding author. Tel.: 1-919-685-9350 x9308, fax: 1-919-685-9360

Email addresses: cstrickland@samsi.info (Christopher Strickland), gerhard@math.colostate.edu (Gerhard Dangelmayr), shipman@math.colostate.edu (Patrick D. Shipman), Sunil.Kumar@colostate.edu (Sunil Kumar),

${ }^{1}$ Current address: Statistical and Applied Mathematical Sciences Institute; 19 T.W. Alexander Drive; Research Triangle Park, NC 27709-4006
} Thomas.Stohlgren@colostate.edu (Thomas J. Stohlgren)
\end{abstract}




\section{Introduction}

The invasion of non-native plants, animals and pathogens has escalated dramatically over the last few decades with the increase of trade, transportation and other elements of globalization (Mack et al., 2000; Evangelista and Kumar, 2011; Stohlgren et al., 2013). Invasive species pose threats to global ecosystems, and they are a major environmental threat of the 21st Century (Mack et al., 2000; Stohlgren and Schnase, 2006). Impacts include loss of native species and habitat, economic suppression, reduced food and water security, and direct threats to human health. For example, the impact of emerald ash borer (Agrilus planipennis) on community residents in Ohio is estimated to be between 1.8 and 7.6 billion for tree loss, removal and replacement (Sydnor et al., 2007); the real estate value of Montana's ranches has been reduced by $40 \%$ due to invasive spotted knapweed (Centaurea maculosa; Sheley et al. (1998)); the glassy winged sharpshooter (Homalodisca vitripennis, an invasive insect which carries a deadly plant bacterium to California grape, raisin, and wine industries and related tourism) is estimated to have an annual cost of approximately $\$ 35$ billion (United States Department of Agriculture (USDA), 2010). Overall economic costs associated with invasive species in the United States are estimated to exceed $\$ 120$ billion per year in terms of control costs, lost productivity, reduced water salvage, and reductions in rangeland quality and property values (Pimentel et al., 2000, 2005). The global economic costs of invasive species are estimated at $\$ 1.4$ trillion annually, representing five percent of the global economy (Keller

et al., 2007; Yemshanov et al., 2009). The economic costs of delaying active control and management efforts would be much greater and probably immeasurable (Eiswerth et al., 2008).

Since control costs rise exponentially with population size, early detection and ecological forecasting of invasive species are urgently needed for rapid response and remain a high priority for resource managers (Crowl et al., 2008). Ecological forecasting requires innovative and flexible mathematical models to map and predict harmful invasive plants, animals, and diseases in time and space (Stohlgren and Schnase, 2006). Consider the difficulty of mapping an invasive species in a non-random environment varying in slope, aspect, elevation, vegetation type, and soil type. Now consider varying rates of spread dependent on optimal versus sub-optimal conditions for reproduc- 
tion and spread. How rapidly, and how far might the species spread in the short- or long-term? What might be the effects of nonlocal spread through a transportation network?

Ecological niche models (also called species distribution models, environmental matching models, and habitat suitability models) are increasingly being used to model and map invasive species distribution potential. Combining statistical algorithms with geographic information systems (GIS), ecological niche models attempt to predict probability of occurrence of a species by using presence-only or presence-absence data in combination with environmental variables to predict the species potential or actual distribution across a landscape (Franklin, 2009; Peterson et al., 2011). These models are based on Hutchinson's classical niche concept: the distributions of species are constrained by biotic interactions (e.g., competition and predation) and abiotic gradients (e.g., elevation, temperature and precipitation) (Hutchinson, 1957).

The spread of species (including invasive species) has been the subject of numerous mathematical models (Higgins and Richardson, 1996, 1999; Higgins et al., 2003; Hastings et al., 2005; Gallien et al., 2010). Many of these models are based on a reaction-diffusion equation with Fickian diffusion and logistic growth (Skellam, 1951; Okubo and Levin, 2001; Maruvka and Shnerb, 2006). Models based on contact-birth processes as introduced by Mollison (1977) represent another approach, in which individuals have fixed spatial locations and the permanent location of each newly born individual is determined by a probability distribution. This approach is better suited to herbaceous invasive plant species that have permanent locations and therefore do not follow Fickian diffusion. In Strickland et al. (2013), we introduced a model, following Mollison's approach (Mollison, 1977), for the time-evolution of the occurrence probability of an invasive plant species. Since the quantity of interest is occurrence probability, not actual population size, data from ecological niche models could be used to determine initial conditions and spatially heterogeneous parameters. Other models combining suitability data with spread are models for the spread of invasive Argentinian ants (Roura-Pascual et al., 2009), and for the spread of invasive plants utilizing a cellular automaton model (Smolik et al., 2010).

Integro-differential equation models that incorporate information about dispersal distribution of plant seeds (Cain et al., 2000) can predict the propagation speed of an invasion front into a landscape (Kot et al., 1996; Strickland et al., 2013). All of these models, however, assume a continuous spatial do- 
main and neglect the role of transportation networks in the spread of invasive species or diseases. Seed dispersal through transportation vectors such as humans (Benninger-Truax et al., 1992), birds (Carlo et al., 2013), or animals (Guiden, 2013) can significantly influence the spread of a species (Harsch et al., 2014), and long-distance jump dispersals have been documented in species such as ants as well (Suarez et al., 2001). Lookingbill et al. (2010) introduced a computational network model to study species spread through fragmented habitats. This approach is based on simulating the random walk of individuals through a landscape graph consisting of nodes representing habitat patches and connections (edges) between nodes. By observing the effect of removing edges, these authors identify bottlenecks in species dispersal. Ferrari et al. (2014) introduced a cellular automaton model for invasive species spread through a landscape graph, allowing the connections between nodes in the network to evolve over time. Simulations of this model point to prominent pathways of spread across a landscape.

In the context of epidemiology, multiple software packages exist to model long range network interactions between geographically distributed metapopulations. For example, GLEAMviz (Balcan et al., 2010) simulates infectious disease spread on a global scale, utilizing detailed airline data and information about local commuter patterns to connect separate metapopulations based on airport locations. The GLEAMviz environment allows the user to specify the exact infection model to be used, as well as transportation rules for each infected compartment (e.g., certain infected, symptomatic classes may not be allowed to travel outside their current occurrence). The Spatial and Temporal Epidemiological Modeling (STEM) (Ford et al., 2006) system is a similar software model, with a greater focus on being open source and modular in its approach. While the large spatial scale of these models makes a metapopulation approach ideal for modeling global or country-wide disease epidemics, it is not particularly adaptable to ecological invasions which are better approximated by a continuous, reaction-diffusion type paradigm on the local level.

The purpose of this paper is to develop a general dynamical systems model for the spread of invasive species or infectious diseases in a discrete (graph-based) network. We describe situations in which both transportation networks and an underlying continuous spread are at play, we then couple the graph-based model to a general model for continuous spread such as the Fisher-KPP equation or a model derived from a contact-birth process.

This paper is organized as follows: In Section 2, we introduce a graph- 
based population model in which individuals can become infected at nodes. The population of the graph is conserved, but every node acts like both a source and sink for individuals to leave and re-enter the graph uninfected. We then conduct analysis on this network model and show how it can be coupled to a spatially continuous infection model to obtain a more complete model of epidemic spread. In Section 3, we extend this model to the case of a herbaceous invader and show numerical results for cheatgrass (Bromus tectorum) spread in Rocky Mountain National Park. We conclude with a discussion of our results in Section 4.

\section{Infectious disease epidemic model}

\subsection{Linear graph model}

To begin modeling long-distance spread, we will first consider the general case of an epidemic with intermediary carrier vectors. These carrier vectors will not be infectious amongst themselves and remain on a transportation network with well defined nodes and directional rates of flow. At each node, carrier vectors can later interact with an underlying, spatially coupled model such as the one developed in Strickland et al. (2013). If the underlying model predicts invader presence at an assigned node location, network carrier vectors will become infected and possibly spread the epidemic to other nodes, which in turn infect the underlying model. The precise mechanics of this concept will be formalized in the following sections, including some immediately relevant analysis.

Consider an individual on a strongly connected ${ }^{2}$, directed graph, and let $X(t)$ be a stochastic variable which gives the node this individual occupies at time $t$. We assume that $X(t)$ satisfies the Markov property and represents a continuous-time Markov chain on the nodes of the graph. Let $g_{i j} \geq 0$ be the transition rate for the $j \rightarrow i$ node edge whenever $i \neq j$, and let

$$
g_{j j}=-\sum_{i \neq j} g_{i j}
$$

We require this value for $g_{j j}$ to assure that the rate at which individuals leave node $j$ is equal to the sum over all the rates for entering destination nodes

\footnotetext{
${ }^{2}$ For every node, there exists a path through the graph to every other node. This is typical for transportation networks.
} 
$i$. In particular, this guarantees that the number of individuals on the graph will be conserved.

Define $\mathbf{p}(t)$ to be the vector of probabilities of finding $X$ at each node at time $t: \mathbf{p}_{i}(t)=\operatorname{Pr}[X(t)=i]$. Since the number of individuals on the graph is conserved, the matrix $G$ such that $(G)_{i j}=g_{i j}$ gives us the evolution of these probabilities on the graph via the first-order equation

$$
\frac{d \mathbf{p}}{d t}=G \mathbf{p}(t) .
$$

Assuming that there are $N$ individuals on the graph and their initial distribution between the nodes is given by the vector $\mathbf{N}(0)=\mathbf{N}_{0}$, we can now set up an initial value problem

$$
\begin{aligned}
\frac{d \mathbf{N}}{d t} & =G \mathbf{N} . \\
\mathbf{N}(0) & =\mathbf{N}_{0}
\end{aligned}
$$

for the expected distribution $\mathbf{N}$ of the individuals over the graph's nodes at time $t$.

The above graph model does not explicitly contain any sources or sinks, but in a localized, real-world transportation network, individuals leave and enter the network from each of the nodes. To incorporate this additional dynamical effect, we will suppose that the number of individuals leaving the graph is balanced by the total number of individuals entering. More specifically, suppose that individuals leave the graph node $i$ with rate $\mu_{i}$ such that $g_{i i} \geq \mu_{i} \geq 0$, and define a vector of these rates, $\boldsymbol{\mu}$. Assuming that the total population on the graph is conserved, individuals also enter the graph at a rate given by $\sum \mu_{i}$ but spread out over the graph via a vector $\boldsymbol{\nu}$, with $\sum \nu_{i}=1$. Then we can define a new matrix of transition rates $H$ by the relations

$$
\begin{aligned}
& h_{i j}=g_{i j}+\left(\boldsymbol{\nu} \boldsymbol{\mu}^{T}\right)_{i j}, \forall i \neq j \\
& h_{j j}=g_{j j}-\mu_{j}+\left(\boldsymbol{\nu} \boldsymbol{\mu}^{T}\right)_{j j},
\end{aligned}
$$

where $\boldsymbol{\nu} \boldsymbol{\mu}^{T}$ denotes the outer product, noting that

$$
h_{j j}=-\sum_{i \neq j} h_{i j}
$$

because $\sum \nu_{i}=1$. 
This definition of $H$ is equivalent to the more shorthand relation $H=$ $G-\operatorname{diag}(\boldsymbol{\mu})+\boldsymbol{\nu} \boldsymbol{\mu}^{T}$. Since the columns of both $G$ and $H$ sum to zero, we are guaranteed that these matrices are singular. While we could simply redefine the entries of $G$ to include the case of $H$, the distinction between these two matrices will prove useful shortly, as we define two distinct subgroups within the graph population $N$.

\subsection{Susceptible/infected vector model on a graph}

Suppose now that all individuals on the graph have the potential to be carriers for some vector-borne disease or invader. We assume that these individuals are unable to contract the disease themselves, at least not in any significant way, or transmit it between members of their own population. Instead, they acquire the disease at certain nodes with a given rate which is variable in time. Define $\beta_{i} \geq 0$ to be the rate of infection at node $i$, and $\boldsymbol{\beta}(t)$ to be the vector of all such rates at time $t$. We will assume that once an individual has the disease, it remains a carrier for all time but may leave the graph as described in the previous section. New members that join the graph are always disease free, but can later become carriers by the same process.

Let $\mathbf{s}$ represent the expected number of susceptible but non-infected individuals at each node, and $\mathbf{c}$ the expected number of infected (carrier) individuals at each node. Using the matrix $G$ and $H$ of transition rates defined in the previous section, we can model the movement of these populations on the graph by the equations

$$
\begin{aligned}
\frac{d \mathbf{s}}{d t} & =\left(H-\operatorname{diag}\left(\boldsymbol{\beta}_{t}\right)\right) \mathbf{s}+(\boldsymbol{\mu} \cdot \mathbf{c}) \boldsymbol{\nu} \\
\frac{d \mathbf{c}}{d t} & =\operatorname{diag}\left(\boldsymbol{\beta}_{t}\right) \mathbf{s}+(G-\operatorname{diag}(\boldsymbol{\mu})) \mathbf{c} \\
\mathbf{s}(0) & =\mathbf{s}_{0} \\
\mathbf{c}(0) & =\mathbf{c}_{0} \\
\text { where } H & =G-\operatorname{diag}(\boldsymbol{\mu})+\boldsymbol{\nu} \boldsymbol{\mu}^{T},
\end{aligned}
$$

and $\operatorname{diag}(\boldsymbol{\mu})$ denotes the diagonal matrix formed from vector $\boldsymbol{\mu}$. With $\boldsymbol{\beta}(t)$ specified, these equations are linear in $\mathbf{s}$ and $\mathbf{c}$ and provide a simple model for disease vectors on a graph which can be subsequently coupled to an underlying epidemic or ecological invasion model, as shown in Sections 2.3 and 3.2 . 
Before continuing, however, there are a few key observations to be made about the model given in Eq. (5) that will give us significant insight into the behavior of our system. First, we rewrite equation (5) in vector form

$$
\begin{gathered}
\frac{d}{d t}\left(\begin{array}{c}
\mathbf{s} \\
\mathbf{c}
\end{array}\right)=A_{t}\left(\begin{array}{c}
\mathbf{s} \\
\mathbf{c}
\end{array}\right) \\
\mathbf{s}(0)=\mathbf{s}_{0} \\
\mathbf{c}(0)=\mathbf{c}_{0}: \text { susceptible }
\end{gathered}
$$

where

$$
A_{t}=\left(\begin{array}{cc}
H-\operatorname{diag}\left(\boldsymbol{\beta}_{t}\right) & \boldsymbol{\nu} \boldsymbol{\mu}^{T} \\
\operatorname{diag}\left(\boldsymbol{\beta}_{t}\right) & G-\operatorname{diag}(\boldsymbol{\mu})
\end{array}\right)
$$

with $\boldsymbol{\nu} \boldsymbol{\mu}^{T}$ once again denoting the outer product. Since the elements of $\boldsymbol{\nu}$ sum to $1, A_{t}$ is a transition matrix of exactly the same general form as $G$ or $H$, with $a_{i j} \geq 0$ for all $i \neq j$ and

$$
a_{j j}=-\sum_{i \neq j} a_{i j}
$$

In fact, we can think of the system as modeling two identical graphs, one for $\mathbf{s}$ and one for $\mathbf{c}$, with specific links between them. Every node of the $\mathbf{s}$ graph is directly reachable from any node of the $\mathbf{c}$ graph through the weighted edges given by $\boldsymbol{\nu} \boldsymbol{\mu}^{T}$, while nodes of the $\mathbf{c}$ graph are only directly connected to corresponding nodes of $\mathbf{s}$ graph when $\beta_{i}>0$. Assuming that at least one $\beta_{i}>0$ and the graph of $\mathbf{s}$ (and thus also $\mathbf{c}$ ) is strongly connected, then the combined graph will also be strongly connected. This observation implies that once a single node is infected, every node becomes susceptible to infection. It also allows us to prove a key theorem (Theorem 1 in the Appendix) about the dynamics of system (6). In the Appendix, we prove that, if the matrix $A$ defined as in equation (7) is formed from a strongly connected graph, and $\boldsymbol{\beta}$ be constant in time, then the asymptotic behavior of any trajectory for the system (6) with initial condition $\left(\mathbf{s}_{0}, \mathbf{c}_{0}\right)$ is that it approaches a stable equilibrium $\left(\mathbf{s}^{*}, \mathbf{c}^{*}\right)$ such that $\sum \mathbf{s}^{*}+\sum \mathbf{c}^{*}=\sum \mathbf{s}_{0}+\sum \mathbf{c}_{0}$.

This theorem will prove useful in the following sections to analyze a variety of combined models. We now introduce the first of these combined models and discuss some of its applications and assumptions. 


\subsection{Coupling the graph with a spatial infection model}

To combine our graph vector model with an underlying model of epidemic spread, let $Y(x, t)$ represent the infected target population (non-graph) over a location space $\Omega$ at time $t$. We will assume that $Y(x, t)$ evolves in time either by a deterministic process

$$
\frac{\partial Y}{\partial t}=F_{1}(Y, x)
$$

or by a stochastic transition probability

$$
\lim _{d t \downarrow 0}\left[\frac{1}{d t} \operatorname{Pr}[Y(x) \rightarrow Y(x)+1 \text { in }(t+d t]]\right]=F_{2}(Y, x)
$$

in absence of the graph and its carrier vectors. To couple the graph to this model, define a vector-valued function $\mathbf{w}(x)$ where the component $w_{i}(x)$ of $\mathbf{w}(x)$ is centered at the location $x_{i} \in \Omega$ corresponding to the location of the node $i$. This allows graph vectors to grow the infected population near assigned locations of the graph nodes, specifically by distributing the infection rate through the distribution $\mathbf{w}(x)$.

We now let

$$
\frac{\partial Y}{\partial t}=F(Y, x, r \mathbf{w}(x) \cdot \mathbf{c})
$$

where $r$ is the infection rate of a graph disease vector. For example, we may take

$$
F(Y, \mathbf{c}, x)=F_{1} \text { or }{ }_{2}(Y, x)+r \mathbf{w}(x) \cdot \mathbf{c},
$$

Finally, given $F$, we explicitly define $\boldsymbol{\beta}(t)$ on the graph using the number of infected individuals $Y$ located around each node. Let $\gamma$ be the rate that an individual from $Y$ infects a disease vector on the graph when contact is made. Then

$$
\begin{aligned}
\beta_{i}(Y) & =\gamma \int_{\Omega} Y(x) w_{i}(x) d x \\
\boldsymbol{\beta}(Y) & =\left\{\beta_{i}(Y)\right\}_{i=1}^{n}
\end{aligned}
$$

Assuming (without loss of generality) that the underlying infection model 
is deterministic, our complete, coupled model can now be written as

$$
\begin{aligned}
\frac{d \mathbf{s}}{d t} & =[H-\operatorname{diag}(\boldsymbol{\beta}(Y))] \mathbf{s}+\left(\boldsymbol{\nu} \boldsymbol{\mu}^{T}\right) \mathbf{c} \\
\frac{d \mathbf{c}}{d t} & =\operatorname{diag}(\boldsymbol{\beta}(Y)) \mathbf{s}+(G-\operatorname{diag}(\boldsymbol{\mu})) \mathbf{c} \\
\frac{\partial Y}{\partial t} & =F(Y, x, r \mathbf{w}(x) \cdot \mathbf{c}) \\
\boldsymbol{\beta}(Y) & =\gamma \int_{\Omega} Y(x, t) \mathbf{w}(x) d x \\
\mathbf{s}(0) & =\mathbf{s}_{0}, \quad \mathbf{c}(0)=\mathbf{c}_{0}, \quad Y(x, 0)=Y_{0}(x) .
\end{aligned}
$$

\section{Invasive species model: multiple time-scales}

\subsection{Underlying assumptions}

The model represented in equation (10) relies on some underlying assumptions that make it best suited for modeling the spread of an infectious disease rather than an herbaceous, biological invader. These include the following:

1. Only one timescale is involved - the infection grows at a rate comparable to movement on the graph.

2. There is no significant latency between contact with an infected graph vector and full infection of a $Y$ individual.

3. Graph coupling not only provides for non-local spread of the disease via graph edges, but also facilitates local infection. Infected individuals $Y$ can infect susceptible vectors $\mathbf{s} \rightarrow \mathbf{c}$ at a node, and the newly infected vectors $\mathbf{c}$ may add to the infection rate at their node of origin before moving on.

While Assumption 3 may be appropriate for an herbaceous invader, the first two assumptions certainly are not. Plants do not germinate, grow, and release new seeds (a year-long process) in the same amount of time as an individual would typically visit a park (a day-long process). Similarly, since infection of a location corresponds to the presence of a seed bearing invasive plant in this context, there is a certain amount of latency between the time seeds are distributed by graph vectors and the resulting infection of a location. To account for this change of context, our model needs to be modified. 


\subsection{Modifying the model for an herbaceous invader}

To address assumptions 1 and 2, we begin by requiring that $F(Y, x)$ be scaled appropriately by some value $\epsilon, 0<\epsilon<<1$. This scaling represents the idea that our model encompasses two timescales - one on which individuals on the graph move from node to node $(\sim t)$ and another on which plants reproduce and grow $(\sim \epsilon)$. Additionally, we will introduce a new variable $L(x, t)$, $x \in \Omega$, representing latent seeds which have been spread by graph vectors but have not yet established themselves in their location. The function $L(x)$ increases from the graph as $Y(x)$ previously did, with members leaving $L(x)$ to $Y(x)$ at a rate of order $\epsilon$. The resulting system can be represented by the equations

$$
\begin{aligned}
\frac{d \mathbf{s}}{d t} & =(H-\operatorname{diag}(\boldsymbol{\beta}(Y))) \mathbf{s}+\left(\boldsymbol{\nu} \boldsymbol{\mu}^{T}\right) \mathbf{c} \\
\frac{d \mathbf{c}}{d t} & =\operatorname{diag}(\boldsymbol{\beta}(Y)) \mathbf{s}+(G-\operatorname{diag}(\boldsymbol{\mu})) \mathbf{c} \\
\frac{\partial L}{\partial t} & =r \mathbf{w}(x) \cdot \mathbf{c}-\sigma L(x, t) h(Y, x)-\delta L \\
\frac{\partial Y}{\partial t} & =\epsilon F(Y, x)+\sigma L(x, t) h(Y, x) \\
\boldsymbol{\beta}(Y) & =\gamma \int_{\Omega} Y(x, t) \mathbf{w}(x) d x \\
\mathbf{s}(0) & =\mathbf{s}_{0}, \quad \mathbf{c}(0)=\mathbf{c}_{0}, \quad Y(x, 0)=Y_{0}(x), \quad L(x, 0)=L_{0}(x)
\end{aligned}
$$

where $\sigma \sim \delta \sim \epsilon$, and $\delta \geq 0$ is a decay term for the latent seeds, and $h(Y, x)$ is a crowding term.

\subsection{Numerical results: Rocky Mountain National Park}

Using the system (11) and the deterministic presence probability model of Strickland et al. (2013), we test our model's performance on predicting the spread of Bromus tectorum, also known as downy brome or cheatgrass, in Rocky Mountain National Park. Bromus tectorum is an introduced annual grass that has infested more than 40 million hectares in the United States (Mack, 1981). Impacts include damage to rangeland, winter crops, hayfields, pastures, grass seed fields, and native shrub species due to increased fires (DiTomaso, 2000). In particular, there is an active B. tectorum invasion taking place in Rocky Mountain National Park, for which presence data exists intermittently since 1996 (Bromberg et al., 2011). Evangelista et al. 
(2008) examined the performance of various ecological niche models including MaxEnt (Phillips et al., 2004, 2006) for predicting potential distribution of B. tectorum in the western United States. Bromberg et al. (2011) used the MaxEnt model for predicting potential suitability for $B$. tectorum using latest Bromus presence data for Rocky Mountain National Park.

We first introduce the probability model of Strickland et al. (2013) with spatial heterogeneity matched to available suitability data for B. tectorum. This model reads

$$
\frac{\partial u(x, t)}{\partial t}=r[J(u(y)) * w(y)](1-u)
$$

where the asterisk denotes the spatial convolution between the two functions. The quantity of interest is the presence probability $u(x, t)$ of the species, the function $w(y)$ is a dispersal kernel, and

$$
J(u)=-\alpha(K) \ln (1-u)+\beta(K) u,
$$

where $K$ is the carrying capacity, and the nonlinear functions $\alpha(K)$ and $\beta(K)$ were determined by comparing numerical simulations of (12) with pseudorandom realizations of the transition probability from which the model was derived.

Dispersal distributions of $B$. tectorum can vary depending on site conditions, including burn history (Monty et al., 2013). However, allowing the distribution kernel $w(y)$ of (12) to vary spatially represents a rather difficult numerical challenge, both in terms of implementation (due to the size of the problem) and the availability of data to parameterize the spatial heterogeneity. Instead, we uniformly utilized a multivariate Laplace distribution (Eltoft et al., 2006) with parameters based on a mean of $(0,0)$, variance of $(1,1)$, and correlation 0 to roughly match the distribution data gathered in Monty et al. (2013) for burned sites. The multivariate Laplace distribution was specifically chosen for its heavy tails and exponential shape, with most of the probability being contained inside a circle of radius 1 from the origin. While this choice certainly represents a worse-case scenario for B. tectorum spread, our modeling efforts are centered around calculating an upper bound on presence probability, and since MaxEnt suitability modeling often includes layers with terrain cover information, we might expect this distribution to be automatically damped in forested areas due to a suitability change.

Fig. 1 displays the solution of the presence probability model (12) for 1999 based on initial data from 1996. The model growth rate was set to 


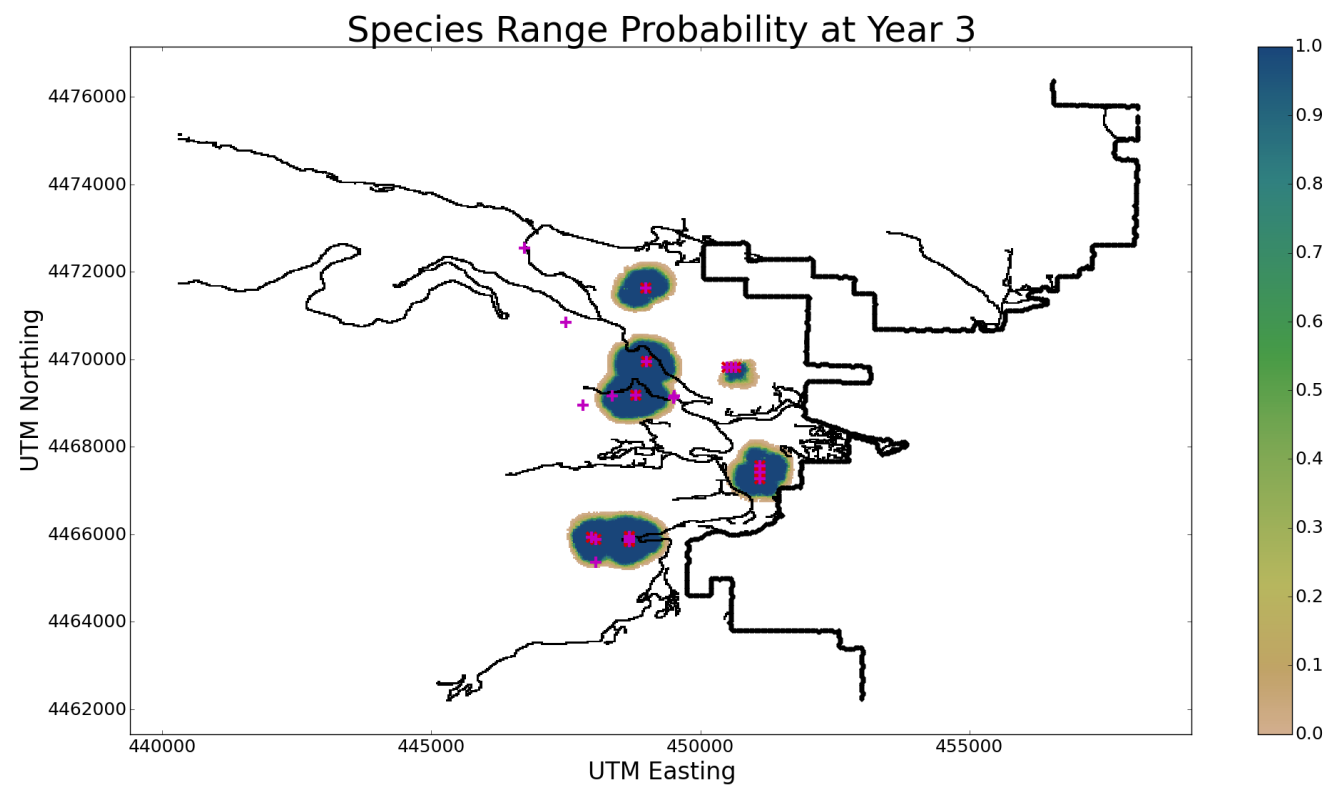

Figure 1: Predicted Rocky Mountain National Park cheatgrass presence for 1999 based on data from 1996 (X marks, overwritten with + marks) using (12). Presence data for 1999 is also plotted (pink + marks). Parameters: $r=5, w \sim$ Laplace $(\mu=(0,0), \sigma=(1,1), \rho=0)$, $K=323$. 
$r=5$, and the carrying capacity was chosen to be $K=323$, based on the number of square feet of cheatgrass necessary to cover 30 square meters (1 square foot of cheatgrass was arbitrarily chosen as the minimal plant unit for establishing presence).

Upon inspection of Fig. 1, one can immediately see that while the model does a fairly good job of capturing local spread with these parameters, other points are completely missed, occurring in locations nowhere close to the model output. While this may be due to a lack of search in these new areas during the 1996 census, it is well documented that trail corridors function as habitat and conduits for movement of plant species in Rocky Mountain National Park (Benninger-Truax et al., 1992) and with the number of visitors $(3,128,446$ per year $)$, it is highly probable that these findings extend to nonlocal movement along paved roads as well. Bromus tectorum seeds can easily attach to clothing, and visitors would find it easy to unknowingly transport propagules down trails or to distant sites using motor vehicles. Note that the additional points in Fig. 1 are conspicuously near roads or trails.

Given that the deficiencies of the model (12) appear to be the result of not capturing long-distance events associated with roads and trails, we now combine this model with the system (11). The 1996-1999 model results and presence data seen in Fig. 1 were used to parameterize the growth rate of (12) while assuming a Laplace distribution for the dispersal kernel $\mathbf{w}(x)$.

Nodes were chosen based on remote sensing data of the park made available through Google Earth (Google, Inc., 2013), including trail heads, trail junctions, parking lots, campgrounds, picnic areas, waterfalls, stables, and ranger stations. Since traffic rates between each of the points was unavailable, flow between graph nodes was estimated using some basic knowledge of the park's attractions, geographical proximity of the nodes, and the function of the nodes in question (e.g., if one has just finished hiking at a trail head, they are less likely to immediately hike another trail - perhaps opting instead to eat lunch at a picnic site). Similar methods were used to estimate the vector $\boldsymbol{\nu}$ for graph entry rates, and vector $\boldsymbol{\mu}$ for graph exit rates.

Since presence probability was used instead of an explicit model for $Y(x, t), \boldsymbol{\beta}(Y)$ was calculated slightly different than described in Section 2.2. The exact equation used was

$$
\boldsymbol{\beta}(\operatorname{Pr}[Y(x, t)>0])=\gamma \int_{\Omega} \operatorname{Pr}[Y(x, t)>0] m(x) \mathbf{w}(x) d x,
$$

where $m(x)$ is the suitability of location $x$ as parameterized by the ecological 


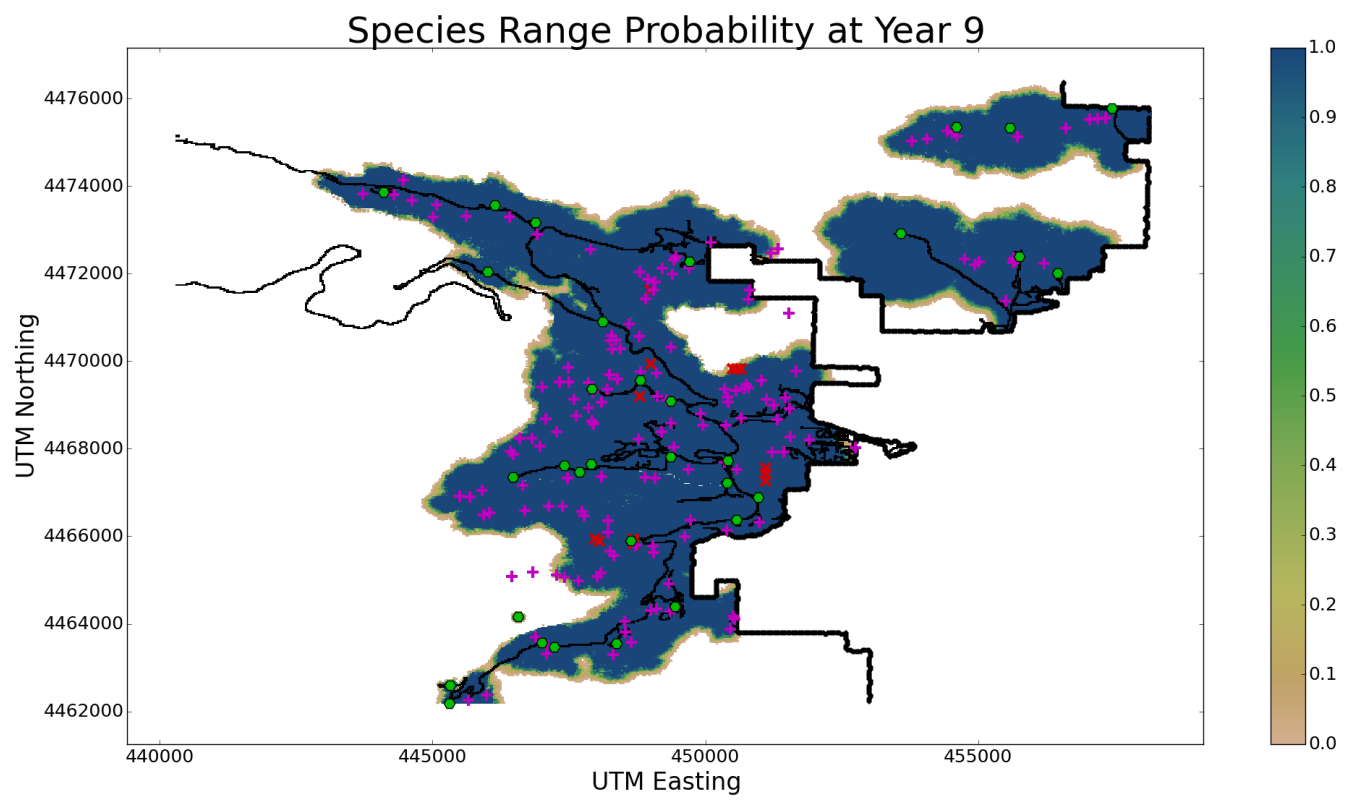

Figure 2: Predicted probability of presence for B. tectorum in Rocky Mountain National Park, based on 1999 presence data using (11) combined with (12). pink + marks are 2008 presence data, green circles are node locations, and red X marks are the 1999 initial presence points.

niche modeling program MaxEnt (Phillips et al., 2004) and $\mathbf{w}(x)$, the distribution function connecting each spatial node location to the graph, was chosen to be a normal distribution with mean $(0,0)$, standard deviation $(5,5)$, and correlation 0 , centered around each of the nodes. The parameter $\gamma$ was arbitrarily chosen to be 0.1 , so that if cheatgrass is present with a probability of 1 and suitability of 1 , then the infection rate onto visitors' clothing is $10 \%$.

Other parameters were chosen as follows: graph infection rate $r=4$, carrying capacity $K=350, \epsilon=1 / 180$ (180 active days in the year, due to winter), $\sigma=\epsilon, \delta=0.33$, and the total number of individuals on the graph at any time $N=5000$ (arbitrarily chosen). The model was run until 2008, starting with data from 1999 and assuming a standard normal probability distribution for $\operatorname{Pr}[Y(x, t)>0]$ around presence points. The results are shown in Figures 2 and 3.

As shown in Figure 2, the graph coupled presence probability model captures all of the 2008 presence locations, with the exception of a couple of 


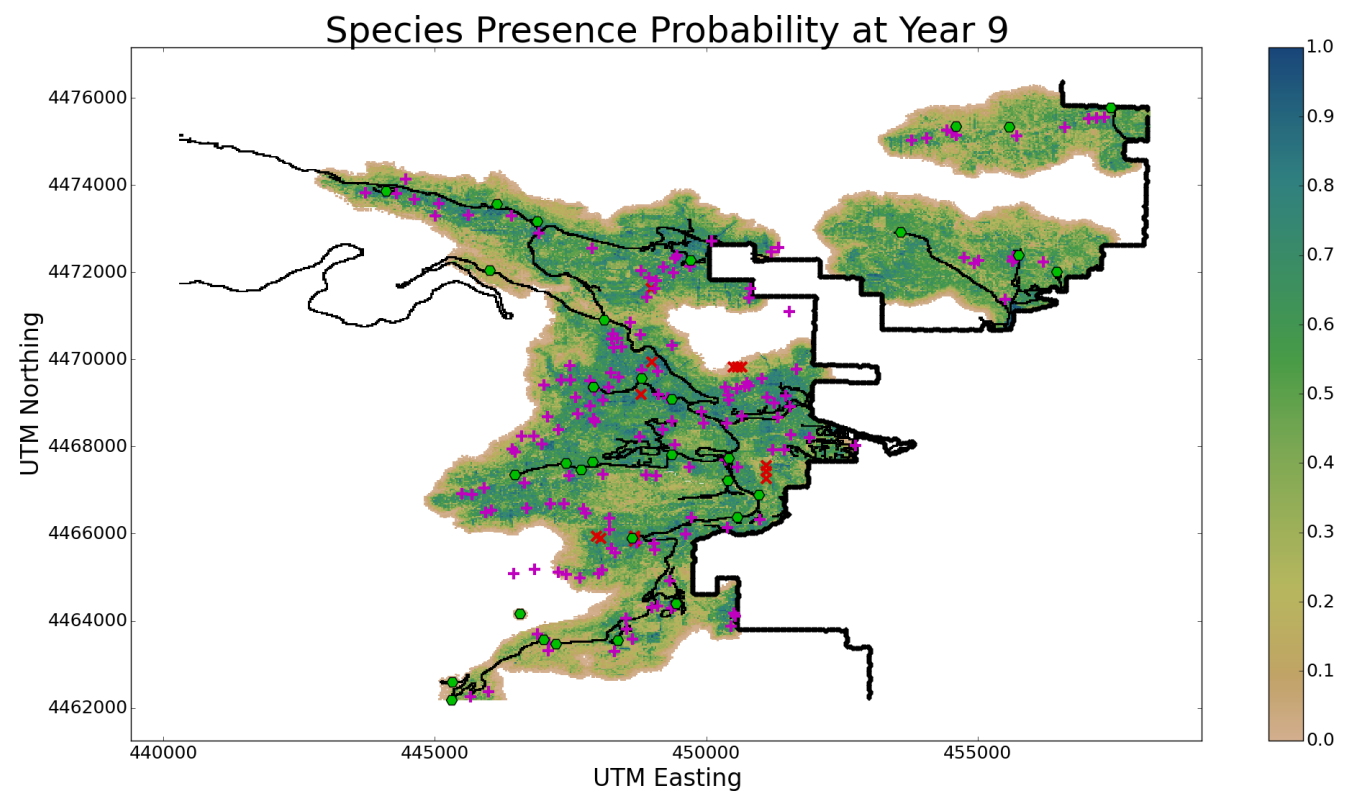

Figure 3: Predicted probability of presence for B. tectorum in Rocky Mountain National Park, scaled by suitability. pink + marks are 2008 presence data, green circles are node locations, and red X marks are the 1999 initial presence points. 
points in the lower left and one nearby the park boundary toward the center of the Figure. Both these locations illustrate certain limitations to the model. The two points in the lower left lie some distance down a trail (not shown), but not near a trailhead or junction included as a network node. Since the combined model only incorporates human movement via the network in this example, the model did not spread the invader fast enough to reach these points. Human facilitated spread off the network (e.g. by non-vehicular movement down trails) can be incorporated using a spatially dependent dispersal kernel, but given the favorable performance of the model in nearly all other locations, we chose not to pursue such an extension in this study. In the other case, the one missed point near the center lies near the boundary of the park, outside of which no presence information was available. Since the town of Estes Park lies just outside the boundary, which includes populations of cheatgrass, it is reasonable to expect that this missed presence point was due to invasion from outside Rocky Mountain National Park boundaries.

Also of interest: just below the two missed points in the lower left of Figure 2 there is a small area of presence probability surrounding a graph node connection, but otherwise disconnected from the main mass of species presence. This node was located at a trail junction close to a lake that could possibly see a lot of travel, but because of the low suitability of the area, the invasion has been largely contained. The lack of model propagation at this node demonstrates the ability of the model to respond when, even though a location is connected to the invasion via the transportation network, the heterogeneity of the terrain makes it difficult for the species to persist locally.

Another feature of the model to note is that in one spot, in the top right of Figure 2, there is a relatively large area of high presence probability but no 2008 presence data. This area was very sparsely sampled in 2008, and while presence was not recorded, one cannot assume from data that the species was entirely absent either. Our model attempts to simulate the spread of potential presence, and not presence in situ. Even within the invasion front, sites will typically consist of areas in which invasive growth is not possible, such as under heavy tree cover. Due to the size of the area to be sampled, Bromus presence was tested only at randomized locations to obtain the presence location data shown in Figure 2 and Figure 3 due to As a result, it is expected for species absence to be recorded in all areas given the heterogeneity of the terrain.

A consequence of randomized sampling is that performance for this study should be judged primarily by its inclusion of presence locations, though 
with additional time and resources, the scaled model in Figure 3 suggests more likely presence locations to visit for further sampling and subsequent validation of the model against overestimation. The result in Figure 3 is exactly the same as in Fig. 2, but it has been scaled by the provided MaxEnt suitability data. One can immediately see the usefulness of this view, as presence data points are most often found in locations that are both highly suitable and predicted to have presence by the model. The Python code used to generate all of these model results has been made available open source at https://github.com/mountaindust/EponaGC.

\section{Discussion}

Using the B. tectorum invasion of Rocky Mountain National Park as an example, we have introduced with equation (10) and (11) a simple, linear model for invasive spread on a transportation network through the action of carrier vectors. Biological invasions rarely occur without human interaction, and understanding long distance dispersal events is critical for accurately assessing the exposure of remote locations to short-term infection. By using the natural graph structure of a transportation network, we can begin to model the probability of spread to remote locations, informing the steps needed to control the invasion.

In addition to the results shown here, we see relevance of our model on a continental or even global scale, where the transportation network takes the form of commercial shipping routes or airline connections. The modular form of equations (10) and (11) should allow for simple implementation with existing spatial models in both epidemiological and ecological settings. We are extending the model to include control, and with an optimal control available, management can quickly prioritize resources to the affected nodes for possible containment. Similarly, we envision applications on a microbiological scale as well, including applications to cancer tumor growth and possible spread to other organs.

We have much to learn about modeling invasive organisms in space and time (Ford et al., 2006; Evangelista et al., 2008; Gallien et al., 2010; Balcan et al., 2010; Bromberg et al., 2011). Many complicated factors regarding long-range dispersal must be incorporated into such models (Cain et al., 2000; Carlo et al., 2013; Strickland et al., 2013). Future patterns of land use change (Higgins et al., 2003), fragmentation, and responses from fires (Monty

et al., 2013) and insect outbreaks and other disturbances may accelerate the 
invasion of cheatgrass reported here. Much like Lookingbill et al. (2010), we see network theory as a way to accommodate complex patterns of invasion. Network approaches, when combined with species distribution models (e.g., Smolik et al. (2010)) may be particularly useful in predicting invasions.

Acknowledgements: S. Kumar was partially funded by the United States Geological Survey and the USDA UV-B Monitoring and Research Program, NREL, Colorado State University, USDA-NRI, 2008-35615-04666. T. Stohlgren was partially supported by CSREES/NRI \#COL0-2008-04348 and Agriculture and Food Research Initiative Competitive Grant no. 2013-68005-21298 from the USDA National Institute of Food and Agriculture. 


\section{Appendix A. Asymptotic behavior of trajectories}

We showed in Section 2.2 that the graph underlying the system (6) is strongly connected. In this appendix, we use this observation to prove the result quoted in Section 2.2 on the asymptotic behavior of trajectories of the system (6).

Lemma 1. Let $G$ be a matrix of transition rates for a strongly connected, directed graph, so that $g_{i j} \geq 0$ when $i \neq j$ and

$$
g_{j j}=-\sum_{i \neq j} g_{i j}<0 .
$$

Then the spectrum of $G$ is equal to $\left\{0, \lambda_{1}, \ldots, \lambda_{\operatorname{dim}(G)-1}\right\}$ where $\lambda_{i}<0$.

Proof. We will proceed by applying the Perron-Frobenius theorem to a linear transformation of $G$, and then interpreting the result for our original matrix. Let $0<\varepsilon<1 / \max \left\{\left|g_{i j}\right|\right\}$, and define a matrix $P_{\varepsilon}=\varepsilon G+I$. Then $P_{\varepsilon}$ is a column stochastic matrix - e.g., all elements of $P_{\varepsilon}$ are between 0 and 1 and the columns of $P_{\varepsilon}$ each sum to 1 . This fact implies that the largest eigenvalue of $P_{\varepsilon}$ is 1 , and that this is also the spectral radius. Furthermore, since the graph associated with $G$ is strongly connected and the positive elements of $P_{\varepsilon}$ exactly correspond to the nonzero elements of $G, P_{\varepsilon}$ is also associated with the strongly connected directed graph, but in the discrete-time sense (more precisely, $P_{\varepsilon}$ is the transition matrix of a strongly connected, discretetime Markov chain). Thus $P_{\varepsilon}$ is an irreducible matrix, and since it is also non-negative, by the Perron-Frobenius theorem we can conclude that the maximal eigenvalue of 1 is unique. Now, since $\varepsilon G=P_{\varepsilon}-I$, it follows that $\varepsilon G$ has a unique largest eigenvalue of 0 , and therefore so does $G$.

Theorem 1. Let the matrix $A$ be defined as in equation (7) from a strongly connected graph and let $\boldsymbol{\beta}$ be constant in time. Then $A$ has a one-dimensional kernel with all other eigenvalues negative. The asymptotic behavior of any trajectory of the system (6) with initial condition $\left(\mathbf{s}_{0}, \mathbf{c}_{0}\right)$ is that it approaches a stable equilibrium $\left(\mathbf{s}^{*}, \mathbf{c}^{*}\right)$ such that $\sum \mathbf{s}^{*}+\sum \mathbf{c}^{*}=\sum \mathbf{s}_{0}+\sum \mathbf{c}_{0}$.

Proof. Suppose that at least one $\beta_{i}>0$. Then by construction of $A$ and our previous observation concerning strongly connected graphs for $\mathbf{s}$ and $\mathbf{c}, A$ is a matrix of transition rates for a strongly connected, directed graph that satisfies the requirements of Lemma 1 . Thus $A$ has a one-dimensional kernel with all other eigenvalues negative. 
Writing out $A$ as

$$
A=\left(\begin{array}{cc}
G-\operatorname{diag}(\boldsymbol{\mu})+\boldsymbol{\nu} \boldsymbol{\mu}^{T}-\operatorname{diag}(\boldsymbol{\beta}) & \boldsymbol{\nu} \boldsymbol{\mu}^{T} \\
\operatorname{diag}(\boldsymbol{\beta}) & G-\operatorname{diag}(\boldsymbol{\mu})
\end{array}\right),
$$

we note that the column sums of $G$ equal 0 , and since $\sum \nu_{i}=1$, the column sums of $\boldsymbol{\nu} \boldsymbol{\mu}^{T}$ equal $\boldsymbol{\mu}$. Thus the column sums of $A$ are zero, which implies balance in the system of differential equations (6) and therefore conservation for the sum of the elements of $\mathbf{s}$ and $\mathbf{c}$ for all time $t$. Thus the solution of the initial value problem approaches the kernel of $A$ at a unique point, where the element sum of an eigenvector associated with the zero eigenvalue is equal to the sum over all the elements of $\left(\mathbf{s}_{0}, \mathbf{c}_{0}\right)$. Since all other modes have negative eigenvalues, they will decay, and the solution will asymptotically approach a stable equilibrium.

Now suppose instead that $\boldsymbol{\beta}$ is equal to the zero vector. Then $A$ can be written as a block upper triangular matrix

$$
A=\left(\begin{array}{cc}
H & \boldsymbol{\nu} \boldsymbol{\mu}^{T} \\
0 & G-\operatorname{diag}(\boldsymbol{\mu})
\end{array}\right) \text {, where } H=G-\operatorname{diag}(\boldsymbol{\mu})+\boldsymbol{\nu} \boldsymbol{\mu}^{T} .
$$

Recall that $G$ is the transition matrix for a continuous-time Markov process on a strongly connected graph. By Lemma 1, $G$ has a unique largest eigenvalue of 0 .

Assume first that $\boldsymbol{\mu}$ is the zero vector. Then $A$ is in fact block diagonal, and represents two completely uncoupled, continuous-time graph processes, both with associated transition matrix $G$. Since the column vectors of $G$ all sum to zero, the differential equation system (6) is balanced and the sum of its solution vector is conserved. Along with the system decoupling, this implies that both $\mathbf{s}$ and $\mathbf{c}$ separately have families of unique, stable steady states, and thus the combined system has one unique, stable steady state for $(\mathbf{s}, \mathbf{c})$ with initial condition $\left(\mathbf{s}_{0}, \mathbf{c}_{0}\right)$.

Now assume that $\boldsymbol{\mu}$ has at least one positive entry. Since $A$ is block upper triangular, the equation for $\frac{d \mathbf{c}}{d t}$ lacks a coupling term,

$$
\frac{d \mathbf{c}}{d t}=(G-\operatorname{diag}(\boldsymbol{\mu})) \mathbf{c} .
$$

We claim that the kernel of $(G-\operatorname{diag}(\boldsymbol{\mu}))$ is trivial. To prove this assertion, we first note that the eigenvalues of both $G$ and $-\operatorname{diag}(\boldsymbol{\mu})$ are non-positive, since $G$ has a unique maximal eigenvalue of 0 and $\boldsymbol{\mu}$ is always non-negative. 
Thus, for any vector $\mathbf{v} \in \operatorname{ker}(G-\operatorname{diag}(\boldsymbol{\mu})), \mathbf{v} \in \operatorname{ker}(G)$ and $\mathbf{v} \in \operatorname{ker}(\operatorname{diag}(\boldsymbol{\mu}))$. Suppose that $\mathbf{v} \in \operatorname{ker}(\operatorname{diag}(\boldsymbol{\mu}))$. Since $\operatorname{diag}(\boldsymbol{\mu})$ is a diagonal matrix with at least one positive entry, $\mathbf{v}$ must have at least one zero element, specifically in the row $k$ corresponding to $\mu_{k} \neq 0$. Now suppose that $\mathbf{v}$ has at least one non-zero element as well.

By construction of $G$, including equation (2) for the time evolution of probabilities through a Markov process on a graph, it is easy to see through Lemma 1 that if $\mathbf{v} \in \operatorname{ker}(G)$, either $v_{i} \geq 0$ for all $i$, or $v_{i} \leq 0$ for all $i$. Since $g_{i j} \geq 0$ whenever $i \neq j, g_{i i}<0$, and at least two entries of any row are nonzero, it follows that in any row $k$ of $G$ such that $v_{k}=0$, there must be nonzero entries only in columns $j$ such that $v_{j}=0$ as well, because all other entries have the same sign. Since $G$ is a matrix of rates on a directed graph, this fact implies that the associated nodes $j$ are only connected to each other. In the case that $\mathbf{v}$ has at least one nonzero element, this observation contradicts the assumption that the graph represented by $G$ is strongly connected. Thus $\mathbf{v}$ must be the zero vector, and we have proven our claim.

With this result, we can now conclude that $\mathbf{c}$ has a single, stable steady state at $\mathbf{c}=0$. We now need only to examine the behavior of $\mathbf{s}$ at this steady state. When $\mathbf{c}=0$, we have

$$
\frac{d \mathbf{s}}{d t}=H \mathbf{s},
$$

and since the graph representation of $H$ is equivalent to that of $G$ up to a change of variables, we can apply Lemma 1 to show that $H$ has a unique largest eigenvalue of 0 . Since the sum of the elements in $\mathbf{s}$ and $\mathbf{c}$ are conserved, and $\mathbf{c}=0$ at the steady state, the kernel of $H$ intersects the solution space of $\mathbf{s}$ at exactly the point where the element sum of $\mathbf{s}$ is equal to the combined sum of the elements of $\mathbf{s}_{0}$ and $\mathbf{c}_{0}$. Thus $(\mathbf{s}, \mathbf{c})$ has exactly one stable steady state solution, which concludes the proof of the theorem. 
Balcan, D., Gonçalves, B., Hu, H., Ramasco, J. J., Colizza, V., Vespignani, A., 2010. Modeling the spatial spread of infectious diseases: The GLobal Epidemic and Mobility computational model. J. Comput. Sci. 1, 132-145. URL http://www.gleamviz.org

Benninger-Truax, M., Vankat, J. L., Schaefer, R. L., 1992. Trail corridors as habitat and conduits for movement of plant species in Rocky Mountain National Park, Colorado, USA. Landsc. Ecol. 6 (4), 269-278.

Bromberg, J. E., Kumar, S., Brown, C. S., Stohlgren, T. J., 2011. Distributional changes and range predictions of downy brome (bromus tectorum) in rocky mountain national park. Invas. Plant Sci. Manag. 4 (2), 173-182.

Cain, M. L., Milligan, B. G., Strand, A. E., 2000. Long-distance seed dispersal in plant populations. Am. J. Bot. 87 (9), 1217-1227.

Carlo, T. A., Garcia, D., Martinez, D., Gleditsch, J. M., Morales, J. M., 2013. Where do seeds go when they go far? Distance and directionality of avian seed dispersal in heterogeneous landscapes. Ecology 94 (2), 301-307.

Crowl, T. A., Crist, T. O., Parmenter, R. R., Belovsky, G., Lugo, A. E., 2008. The spread of invasive species and infectious disease as drivers of ecosystem change. Front. Ecol. Environ. 6, 238-246.

DiTomaso, J. M., 2000. Invasive weeds in rangelands: Species, impacts, and management. Weed Sci. 48, 255-265.

Eiswerth, M., Johnson, W., Lewis, S., Hughes, L., 2008. The economic costs of delaying invasive weed control: An illustration based on Nevada's tall whitetop initiative. University of Nevada, Cooperative Extension, Reno, Nevada.

Eltoft, T., Kim, T., Lee, T., 2006. On the multivariate laplace distribution. IEEE Signal Proc. Let. 13 (5).

Evangelista, P., Kumar, S., 2011. Trade and transportation is changing the game. Curr. Zoology 57 (5), II.

Evangelista, P. H., Kumar, S., Stohlgren, T. J., Jarnevich, C. S., Crall, A. W., Norman III, J. B., Barnett, D. T., 2008. Modelling invasion for a habitat generalist and a specialist plant species. Diversit. Distrib. 14, 808-817. 
Ferrari, J. R., Preisser, E. L., Fitzpatrick, M. C., 2014. Modeling the spread of invasive species using dynamic network models. Biol. Invasions 16 (4), 949-960.

Ford, D. A., Kaufman, J. H., Eiron, I., 2006. An extensible spatial and temporal epidemiological modelling system. Int. J. Health Geogr. 5 (4).

URL http://www.eclipse.org/stem/

Franklin, J., 2009. Book. Cambridge University Press, Cambridge, UK.

Gallien, L., Munkemuller, T., Albert, C. H., Boulangeat, I., Thuiller, W., 2010. Predicting potential distributions of invasive species: where to go from here? Divers. Distrib. 16, 331-342.

Guiden, P. W., 2013. Dispersal of honey locus (Gleditsia triacanthos) seeds by white-tailed deer. Ecol. Res. 31 (4), 356-358.

Harsch, M. A., Zhou, Y., HilleRisLambers, J., Kot, M., 2014. Keeping pace with climate change: Stage-structured moving-habitat models. Am. Nat. 184 (1), 25-37.

Hastings, A., Cuddington, K., Davies, K. F., Dugaw, C. J., Elmendorf, S., Freestone, A., Harrison, S., Holland, M., Lambrinos, J., Malvadkar, U., Melbourne, B. A., Moore, K., Taylor, C., Thomson, D., 2005. The spatial spread of invasions: new developments in theory and evidence. Ecol. Lett. 8, 91-101.

Higgins, S. I., Lavorel, S., Revilla, E., 2003. Estimating plant migration rates under habitat loss and fragmentation. Oikos 101, 354-366.

Higgins, S. I., Richardson, D. M., 1996. A review of models of alien plant spread. Ecol. Model. 87 (13), 249-265.

Higgins, S. I., Richardson, D. M., 1999. Predicting plant migration rates in a changing world: The role of long-distance dispersal. Am. Nat. 153 (5), $464-475$.

Hutchinson, G. E., 1957. Population studies-animal ecology and demography-concluding remarks. In: Cold Spring Harbor Symposia on Quantitative Biology. Vol. 22. pp. 415-427. 
Keller, R. P., Lodge, D. M., Finnoff, D. C., 2007. Risk assessment for invasive species produces net bioeconomic benefits. P. Natl. Acad. Sci. USA 104, 203-207.

Kot, M., Lewis, M. A., van den Driessche, P., 1996. Dispersal data and the spread of invading organisms. Ecology 77 (7), 2027-2042.

Lookingbill, T. R., Gardner, R. H., Ferrari, J. R., Keller, C. E., 2010. Combining a dispersal model with network theory to assess habitat connectivity. Ecol. Appl. 20, 427-441.

Mack, R., 1981. Invasion of Bromus tectorum L. into western north america; an ecological chronicle. Agro-ecosystems 7, 145-165.

Mack, R. N., Simberloff, D., Lonsdale, W. M., Evans, H., Clout, M., Bazzaz, F. A., 2000. Biotic invasions: Causes, epidemiology, global consequences, and control. Ecol. Appl. 10, 689-710.

Maruvka, Y. E., Shnerb, N. M., 2006. Nonlocal competition and logistic growth: patterns, defects, and fronts. Phys. Rev. E 73, 1-12.

Mollison, D., 1977. Spatial contact models for ecological and epidemic spread. J. R. Statist. Soc. 39 (3), 283-326.

Monty, A., Brown, C. S., Johnston, D. B., 2013. Fire promotes downy brome (bromus tectorum 1.) seed dispersal. Biol. Invasions 15, 1113-1123.

Okubo, A., Levin, S. A., 2001. Diffusion and ecological problems. SpringerVerlag, New York, NY.

Peterson, A. T., Soberon, J., Pearson, R. G., Anderson, R. P., MartinezMeyer, E., Nakamura, M., Araujo, M. B., 2011. Ecological Niches and Geographic Distributions. Princeton University Press, Princeton, N.J.

Phillips, S. J., Anderson, R. P., Schapire, R. E., 2006. A maximum entropy approach to species geographic distributions. Ecol. Model. 190 (3-4), 231259.

Phillips, S. J., Dudik, M., Schapire, R. E., 2004. A maximum entropy approach to species distribution modeling. In: Proceedings of the TwentyFirst International Conference on Machine Learning. pp. 655-662. 
Pimentel, D., Lach, L., Zuniga, R., Morrison, D., 2000. Environmental and economic costs of nonindigenous species in the United States. Biosci. 50, $53-65$.

Pimentel, D., Zuniga, R., Morrison, D., 2005. Update on the environmental and economic costs associated with alien-invasive species in the United States. Ecol. Econ. 52, 273-288.

Roura-Pascual, N., Bas, J. M., Thuiller, W., Hui, C., Krug, R. M., Brotons, L., 2009. From introduction to equilibrium: reconstructing the invasive pathways of the Argentine ant in a Mediterranean region. Glob. Change Biol. 15, 2101-2115.

Sheley, R. L., Jacobs, J. S., Carpinelli, M. F., 1998. Distribution, biology, and management of diffuse knapweed (Centaurea diffusa) and spotted knapweed (Centaurea maculosa). Weed Technology 12, 353-362.

Skellam, J. G., 1951. Random dispersal in theoretical populations. Biom. 38 (1), 196-218.

Smolik, M. G., Dullinger, S., Essl, F., Kleinbauer, I., Leitner, M., Peterseil, J., Stadler, L. M., Vogl, G., 2010. Integrating species distribution models and interacting particle systems to predict the spread of an invasive alien plant. J. Biogeogr. 37, 411-422.

Stohlgren, T. J., Pyek, P., Kartesz, J., Misako, N., Pauchard, A., Winter, M., Pino, J., Richardson, D. M., Wilson, J., Murray, B. R., Phillips, M. L., Celesti-Grapow, L., Graham, J., 2013. Globalization effects on common plant species. In: Levin, S. A. (Ed.), Encyclopedia of Biodiversity, 2nd Edition. Academic Press, Waltham, MA, pp. 700-706.

Stohlgren, T. J., Schnase, J. L., 2006. Risk analysis for biological hazards: what we need to know about invasive species. Risk Anal. 26, 163-173.

Strickland, C., Dangelmayr, G., Shipman, P. D., 2013. Modeling the presence probability of invasive plant species with nonlocal dispersal. J. Math. Biol. $332,123-135$.

Suarez, A. V., Holway, D. A., Case, T. J., 2001. Patterns of spread in biological invasions dominated by long-distance jump dispersal: Insights from Argentine ants. P. Natl. Acad. Sci. USA 98 (3), 1095-1100. 
Sydnor, T. D., Bumgardner, M., Todd, A., 2007. The potential economic impacts of emerald ash borer (Agrilus planipennis) on Ohio, U.S., communities. Arboriculture and Urban Forestry 33, 48-54.

United States Department of Agriculture (USDA), 2010. Invasive exotic animals costing U.S. billions of dollars.

URL http://www.invasivespeciesinfo.gov/economic/main.shtml

Yemshanov, D., McKenney, D. W., Pedlar, J. H., Koch, F. H., D, C., 2009. Towards an integrated approach to modelling the risks and impacts of invasive forest species. Env. Rev. 17, 163-178. 